\title{
Sterben erzählen im Spielfilm
}

Pierre Bühler

\section{Zum Einstieg: erzählerisch Abschied nehmen}

Jon Avnets Film Fried Green Tomatoes (USA, 1991) erzählt, wie zwei junge Frauen, Idgie und Ruth, in den 1930er Jahren in Alabama ein Restaurant mit Spezialitäten wie Barbecue und, eben, Fried Green Tomatoes führen. Doch die eine Freundin, Ruth, wird unheilbar krank, was für Idgie unerträglich ist. Seit ihrer Kindheit bereits ist Idgie berühmt für ihre unglaublichen, aus der Phantasie geschöpften Geschichten. Die Todesstunde naht: Ruth liegt im Sterben, Idgie sollte Abschied nehmen, bringt es aber nicht fertig, geht wild im Zimmer herum. Ruth bittet sie darum, ihr noch einmal die schon mehrmals erzählte Geschichte vom Teich und den wilden Enten zu erzählen. Es gebe einen Teich in der Nähe, erzählt nun Idgie, zuerst zögerlich, und dann immer begeisterter, auf dem sich immer wieder wilde Enten auf dem Durchflug ausgeruht hätten. Erzählend wendet sie sich langsam zum Fenster und schaut hinaus. An einem eiskalten Wintertag sei dieser Teich so schnell eingefroren, dass alle Enten im Eis gefangen geblieben seien. Doch dann hätten sie plötzlich wie auf Befehl alle ihre Flügel aufgeschlagen und seien weggeflogen. Das Eis hätte aber so sehr an ihren Federn gehaftet, dass die Enten die ganze Eisschicht, die über dem Teich lag, im Flug weggetragen hätten, so dass der Teich plötzlich wieder ganz eisfrei war. Als sich Idgie wendet, um zu schauen, wie ihre Geschichte auf ihre Freundin gewirkt hat, entdeckt sie, dass diese im Laufe ihrer Erzählung gestorben ist. Die Phantasie ist Wirklichkeit geworden: Beim Erzählen ist die Eisschicht des schweren Abschiednehmens mit den wilden Enten weggeflogen. Das Trauern um die verstorbene Freundin darf beginnen, befreit vom Nicht-Abschied-nehmen-Können.

\section{Filmische Inszenierungen des Sterbens}

Inszenierungen des Sterbens sind in Spielfilmen allgegenwärtig. Da es in ihnen um das Leben der Menschen geht, befassen sie sich auch sehr intensiv mit dem Sterben. In Spielfilmen wird viel gestorben, 
und auf vielfältige Weisen. Das Motiv des Sterbens kann sehr punktuell vorkommen, in Filmen, in denen auch noch viele andere Themen behandelt werden (das ist z.B. in Fried Green Tomatoes der Fall), und es gibt Spielfilme, in denen das Sterben zum zentralen Motiv wird, so dass man regelrecht von Sterbe-Filmen sprechen kann.

Der Tod kann oft plötzlich eintreffen, mehr oder weniger gewaltsam, etwa durch Unfall oder durch Mord. Aber gerade der sequenzielle Charakter des Filmes erlaubt es auch, in der Reihenfolge der Bilder den Prozess des Sterbens darzustellen. Das muss nicht nur linear geschehen, es können Flashbacks oder Vorwegnahmen eingefügt werden, die auch das existentielle Verarbeiten des Prozesses bei der sterbenden Person und den sie begleitenden Mitmenschen thematisieren.

Die bewusst reflektierte Inszenierung erlaubt es auch, mit der bildnerischen Darstellung unterschiedlich umzugehen: Das Sterben kann hautnah, in direkter Bildsprache repräsentiert werden; es kann aber auch in indirekter Kommunikation geschehen, so dass das Sterben selbst nur wenig "ins Bild" hineinkommt. Ruths Sterben, das einleitend geschildert wurde, wird gerade nicht gezeigt: Die Erzählung lenkt davon ab, diesem Sterben zuschauen zu müssen, sowohl für die Freundin Idgie als auch für die Filmzuschauer ...

Die Filminszenierungen können ebenfalls unterschiedliche Deutungsmuster ins Spiel bringen, auch hier wieder mehr oder weniger massiv oder diskret, Deutungsmuster, die es erlauben, vom Sterben Abstand zu nehmen oder es durchzuarbeiten in Hinsicht aufTiefendimensionen, die sich in ihm offenbaren.

Zur Illustration dieser vielfaltigen Arten der filmischen Inszenierung des Sterbens seien kurz drei Filme evoziert, in denen der Prozess mit unterschiedlichen Konnotationen thematisiert wird. Geht es im ersten Spielfilm mehr um eine ästhetische Lebenseinstellung, die im Sterben an ihre Grenze stösst, handelt der zweite mehr von der ethischen Verpflichtung dem Sterben gegenüber. Im dritten schliesslich kommen religiöse Deutungsmotive zum Tragen (deshalb wird dieses dritte Beispiel auch etwas ausführlicher behandelt). ${ }^{1}$

\footnotetext{
1 Nicht ganz zufällig entsprechen die drei ausgewählten Spielfilme den drei Existenzsphären bei Søren Kierkegaard.
} 


\section{Bob Fosses All that Jazz (USA, 1979)}

Dieser Spielfilm ist eine teilweise autobiographische Fiktion: Der Filmemacher thematisiert seine eigene schwierige Situation im Jahre 1975, als er an der Inszenierung des Broadway Musicals Chicago arbeitete, sich für die Edition seines Films über den Stand-up-Komödiant Lenny Bruce einsetzte und zugleich mit grossen Ermüdungs- und Gesundheitsproblemen kämpfte. In der Fiktionalisierung des Filmes ist die Hauptgestalt, Joe Gideon, wie Bob Fosse selbst, Theaterdirektor und Choreograph. Kettenraucher, medikamentenabhängig und sexsüchtig, mit einem total erschöpften Körper kämpfend, arbeitet er unablässig, äusserlich gesehen an der Inszenierung eines Musicals, aber zuerst und vor allem an der Inszenierung seines Lebens. Nach dem Grundprinzip »The show must go on " gestaltet er sein ganzes Leben als eine ständige Show, ganz auf Bühnenerfolg ausgerichtet. Alle um ihn herum raten ihm auszuruhen, auszuspannen, doch kann er es nicht mehr, weil er wie in einem Teufelskreis gefangen ist. Er spürt die Verschlechterung seines Gesundheitszustandes, ahnt die Gefahr des Todes, kann sich ihr aber nicht stellen, sondern flieht vor seiner Sterblichkeit in noch mehr Arbeit, Sex, Medikamente und Zigaretten. Er muss wegen eines Herzinfarkts ins Spital eingeliefert werden, und es wird immer unsicherer, ob er noch fähig sein wird, das Musical zur Aufführung zu bringen. Auch für die Edition seines Filmes bekommt er immer schlechtere Nachrichten. Mehr und mehr versetzt sich Gideon in Halluzinationen hinein, in denen er sich selbst als tanzende Hauptgestalt auf der Bühne inszeniert, so dass sich die Show in seiner Phantasie mehr und mehr zu einem Totentanz entwickelt (er tanzt denn auch mit einem Todesengel, der Tänzerin Angelique). Noch einmal wird er in einem grossen Finale das, was er immer schon sein wollte: der bewunderte Star einer erfolgreichen Show. Die Szene, mit der der Film schliesst, steht jedoch in krassem Widerspruch dazu: Sie zeigt, wie Gideons toter Körper in eine Leichenhülle verpackt wird.

Interessant ist, dass immer wieder, als Ausschnitt aus Joe Gideons Film, eine Nummer in Lennys Stand-up-Komödie eingespielt wird, in der sich dieser über die fünf Etappen der Auseinandersetzung mit dem Sterben gemäss Elisabeth Kübler-Ross lustig macht. Durch diese ironische Distanzierung wird Joe Gideons Sterbeprozess indirekt beleuchtet, denn er muss merken, dass er im Kampf mit seiner Lebenskrise diese fünf Gemütszustände ungewollt durchmachen muss: Stets schwankt er hin und her, zwischen Wut, Verneinung, Verhandlung, Depression und Zustimmung, ohne das Ganze des Prozesses 
wirklich wahrhaben zu wollen, stets vor ihm fliehend. Doch mehr und mehr scheint die Show seines Lebens zu scheitern, was eigentlich nur seine Lust steigert, in einer imaginären Darbietung noch ein letztes Mal ganz vorne zu sein. Mit der Leichenhülle endet diese Show, und die Bühnenlichter gehen aus. Das Ideal eines Lebens als einer unablässigen Parade kommt zu seiner Grenze, obschon Bob Fosse selbst diese Krise von 1975 überlebt (er stirbt dann an einem Herzinfarkt im Jahre 1987).

\section{Cesc Gays Truman (Spanien/Argentinien, 2015)}

Julián und Tomás sind zwei argentinische Männer mittleren Alters, die seit ihrer Jugend befreundet sind. Der erste lebt in Madrid, geschieden und vereinsamt; der zweite lebt, verheiratet und Familienvater, in Kanada. Dieser weiss darum, dass es seinem Madrider Freund, der krebskrank ist, schlecht geht, und so überrascht er ihn mit einem Besuch für ein paar Tage. Seine Absicht ist unter anderem, den Freund dazu anzuhalten, weitere Therapien zu unternehmen, gegen den nahenden Tod anzukämpfen. Doch dieser Versuch scheitert schnell: Julián hat endgültig entschieden, nichts mehr zu unternehmen, sondern sich nun auf sein Sterben vorzubereiten, seine Sachen in Ordnung zu bringen und alles Nötige zu regeln. Jeder stirbt so gut wie er kann, das ist Juliáns Überzeugung, und da nun der Freund aus Kanada da ist, soll er ihm bei seinen Sterbevorbereitungen behilflich sein. Und so wird der kurze Besuch in Madrid zu einer ungeahnten Überraschung für Tomás: Er wird plötzlich ganz konkret, viel konkreter als er es gewünscht hätte, mit dem Sterben konfrontiert, indem er die verschiedenen Abklärungen seines Freundes begleiten muss: den letzten Besuch beim Arzt, um diesem mitzuteilen, dass die Therapien abgebrochen werden; den Besuch im Bestattungsgeschäft, um einen passenden Sarg auszuwählen, usw. Zum "Aufräumen" gehört auch die Verarbeitung der Vergangenheit: Mit Reue schaut Julián auf ein etwas zerrüttetes Leben mit viel Untreue und Betrug, eine gescheiterte Ehe, einen vernachlässigten Sohn (deshalb fliegen die zwei Freunde auch nach Amsterdam, um mit dem dort studierenden Sohn eine Aussöhnung zu vollziehen). In vielen Gesprächen, zwischen tiefer Melancholie und befreiendem Humor schwankend, erleben sie so in Ernst und Gelassenheit zugleich, was ihre letzte gemeinsame Zeit sein wird. Beide wissen um den kommenden Abschied, nehmen aber immer wieder spielerisch davon Abstand. 
Ganz zentral im ganzen Film ist auch Truman, der dem Film seinen Titel verliehen hat: ein alter, riesengrosser Hund, Juliáns jahrelanger treuer Begleiter. Was soll nach Juliáns Tod mit ihm geschehen? Sehr besorgt um seinen Hund (den Tierarzt fragt Julián sogar, ob denn Hunde nach dem Tod ihres Herrn auch trauern) sucht er nach einem neuen Besitzer oder einer neuen Besitzerin, und Tomás muss auch hier seinen Freund bei den Treffen mit den verschiedenen Interessenten begleiten. Irgendwie kommt keine Lösung zustande, und als sich die zwei Freunde am Flughafen verabschieden, kommt alles ganz anders heraus: Julián hat insgeheim alle Billette und Ausreisepapiere für Truman vorbereitet, so dass er mit Tomás nach Kanada fliegen darf! Truman, das treueste Tier, soll mit dem treuesten Menschen weiterleben dürfen. So wird der alte Hund zum Kristallisationspunkt der Freundschaft, besiegelt sie beim schmerzhaften endgültigen Abschied.

\section{Ingmar Bergmans Schreie und Flüstern (Viskningar och rop, Schweden, 1972) ${ }^{2}$}

Die Gestalten in Bergmans Filmen setzen sich mit existentiellen Themen auseinander: Sie kämpfen mit derVereinsamung und leiden an schwierigen zwischenmenschlichen Beziehungen, ringen mit der Perspektive des Todes, fragen nach Schuld und Vergebung, scheitern am Unsinn des Lebens und suchen leidenschaftlich nach Gott.Viele dieser Themen finden sich in Schreie und Flüstern wieder. Der Film spielt auf einem schönen Gutshof am Ende des 19. Jahrhunderts. Erzählt wird, mit unterschiedlichen Flashbacks, ein Abschnitt aus dem Leben vierer Frauen, die auch die Hauptgestalten des Films sind: Agnes, die ältere Schwester, die schwerkrank ist und im Sterben liegt; Maria und Karin, die zwei Schwestern, die zu Besuch gekommen sind, um Agnes in ihrem Leiden und Sterben zu begleiten; Anna, die Magd, die die kranke Agnes pflegt. Der Film beschreibt sowohl das Leiden und Sterben von Agnes als auch die unterschiedlichen Arten der drei anderen Frauen, darauf zu reagieren, darauf einzugehen oder sich davon zu distanzieren.

2 Der Film wird zitiert nach der Filmerzählung in deutscher Übersetzung: Ingmar Bergman, Schreie und Flüstern, in: Bergman, Wilde Erdbeeren und andere Filmerzählungen, München 1977, 403-444. Dieser Abschnitt greift auf gewisse Elemente eines ausführlicheren Aufsatzes zu Schreie und Flüstern zurück, der 2017 in einem von Daria Pezzoli-Olgiati unter dem Titel »Leid-Bilder« herausgegebenen Sammelband zu Passionsfilmen erscheinen wird. 
Nachdem Agnes gestorben ist, kommt der Pfarrer, um die Beerdigung vorzubereiten, und spricht am Sterbebett ein Gebet. Nach der Beerdigung, bevor die zwei Schwestern mit ihren Ehemännern wieder wegziehen, wird die Magd entlassen. Als Andenken wünscht sie sich das Tagebuch von Agnes, aus dem im Film ab und zu in OffStimme gelesen wurde. Auch in der Schlussszene wird eine Passage zitiert, in der Agnes einen Spaziergang mit den Schwestern im Park des Gutshofes beschreibt:

"Ich hatte die Augen ganz fest geschlossen, wollte den Augenblick festhalten und dachte: Das hier ist auf jeden Fall Glück. Ich kann mir nichts Besseres wünschen. Nun darf ich einige Augenblicke Vollkommenheit erleben. Und ich empfinde eine grosse Dankbarkeit gegenüber meinem Leben, das mir so viel gibt. ${ }^{3}$

Bergmans Film ist vielschichtig und kann auf unterschiedlichen Ebenen interpretiert werden. Wir heben hier eine dieser Ebenen hervor: die diskreten metaphorischen Anspielungen auf die Passionsgeschichte Jesu in den neutestamentlichen Evangelien. Verschiedene Hinweise lassen sich bei genauer Betrachtung wahrnehmen:

- Während des ganzen Films ticken die Wanduhren und läuten die Glocken, immer wieder an die laufende Zeit mahnend: Das erinnert stark an die Thematik der Stunden in der Passionsgeschichte: »es war die sechste Stunde, als ...», »siehe, es war die neunte Stunde«.

- Ferner kann der Vorname Agnes ein weiteres Indiz sein: Er kommt vom lateinischen agnus, und verweist damit wahrscheinlich auf das Passionsmotiv des agnus Dei, wie etwa in Joh 1,29 als Zeugnis Johannes des Täufers zum Ausdruck gebracht: "Seht, das Lamm Gottes, das die Sünde der Welt wegnimmt."Im Hintergrund steht auch das griechische hagnos, das "rein«, "unschuldig«, bedeutet, auch in kultischem Sinn. Somit kann Agnes als unschuldiges Opfer interpretiert werden.

Kommen wir nun aber zu drei wichtigeren Aspekten.

- Agnes ist in einem grossen Todesschrei gestorben (wie Jesus bei Markus und Matthäus? ${ }^{4}$. Sie liegt im Bett, es werden ihr die Augen geschlossen, und ihr Leib wird aufgedeckt. Die Beine liegen, wie sie oft in der Kunstgeschichte beim Gekreuzigten dargestellt werden: das eine gerade, das andere angewinkelt. Die Arme waren wie beim

3 Bergman, Schreie und Flüstern, 444.

4 Mk 15,37/Mt 27,50: "Da stiess Jesus einen lauten Schrei aus und verschied." In beiden Evangelien steht in der Nähe dieses Todesschreis ein anderer lauter Schrei des Gekreuzigten, der in der neunten Stunde ausgesprochen wird (Zitat aus Ps 22,1; vgl. Mk 15,34/Mt 27,46): »Mein Gott, mein Gott, warum hast du mich verlassen?» 
Gekreuzigten weit ausgebreitet; sie werden nun zum Leib zurückgebracht und die Hände zusammengefaltet.

An einer entscheidenden Stelle des Films geschieht die christologische Metaphorisierung der Agnes als gesprochene Ergänzung zu den bildnerischen Motiven. Diese explizit religiöse Spur ist mit dem Auftreten des Pfarrers verbunden. Nach den Begrüssungen tritt der Pfarrer ans Sterbebett, um ein Gebet zu sprechen. Auffallend an dieser Szene ist die Aufteilung des Gebets in zwei sehr unterschiedliche Momente. Zunächst spricht der Pfarrer ein liturgisch geprägtes Gebet für die Verstorbene:

"Gott, unser Vater, hat in seiner Allweisheit beschlossen, dich in der Blüte deiner Jugend heimzurufen. Vorher befand er dich für würdig, ein schweres und langwieriges Leiden zu tragen. Du hast dich ihm geduldig unterworfen, ohne zu klagen, in der Gewissheit, dass deine Sünden dir durch den Kreuzestod deines Herrn Jesus Christus vergeben würden. Möge dein Vater im Himmel sich deiner Seele erbarmen, wenn du vor ihn hintrittst. Mögen dich seine Engel der Erinnerung an deinen irdischen Schmerz entheben. $\aleph^{5}$

An dieser Stelle verstummt der Pfarrer plötzlich.Verlegen zögert er - und tritt plötzlich aus seiner Rolle: Er kniet nieder vor Agnes, und die anderen im Raum, ebenfalls verlegen, tun es ihm gleich. Er betet nun nicht mehr für sie, sondern vielmehr $z u$ ihr, ihr würdiges Leiden nun ganz anders aufnehmend, als ein stellvertretendes Leiden.

»Wenn du unser Leiden in deinem armen Körper vereint hast, wenn du es mit dir durch den Tod getragen hast, wenn du Gott dort in dem anderen Lande begegnest, wenn er sein Angesicht dir zuwendet, wenn du dort eine Sprache sprechen kannst, die dieser Gott versteht, wenn du dort sprechen kannst zu diesem Gott ... Wenn es so ist - bitte für uns, Agnes, liebes Kindchen, höre, was ich dir jetzt sage. Bitte für uns, die wir hiergeblieben sind auf dieser dunklen, schmutzigen Erde unter einem leeren und grausamen Himmel. Lege deine Bürde des Leidens Gott zu Füßen und bitte ihn, uns zu begnadigen, bitte ihn, uns endlich zu befreien aus unserer Angst, unserem Überdruss und unserem tiefen Zweifel. Bitte ihn um einen Sinn für unser Leben, Agnes, die du so unfassbar und so lange gelitten hast, du musst würdig sein, für uns zu sprechen. $\otimes^{6}$

5 Bergman, Schreie und Flüstern, 421.

6 Ebd., 422. 
Nachdem der Pfarrer wieder aufgestanden ist, bleibt er einen Augenblick verlegen, spricht davon, dass Agnes seine Konfirmandin war, dass sie viele Gespräche führten und ihr Glaube stärker war als seiner, und verabschiedet sich. Die Wirkung seiner Worte aber bleibt: Durch sein Gebet wurde Agnes zu einer christologischen Figur. Mit all ihrem Leiden soll sie vor Gott unsere Fürbitterin werden und für uns eintreten.

Dieser christologischen Figur gegenüber werden die drei anderen Frauen anhand ihrer Reaktionen auf das Sterben in einer Art Grundtypologie in ihren unterschiedlichen Lebenseinstellungen enthüllt.

In Maria zuerst nimmt man Spuren von Angst wahr, aber zugleich eine schmeichelnde Leichtigkeit, die auch dazu führt, dass sie vor dem Sterben ihrer Schwester immer wieder erschrocken flieht, der eigentlichen Herausforderung mit Ausreden ausweicht. Sie stellt einen Menschen dar, der stets an der Oberfläche des Lebens bleibt, immer wieder ins Unbestimmte flieht, weil er stets nach dem Prinzip der Lust lebt, ganz in der Nähe dessen, was Joe Gideon in Bob Fosses Spielfilm vertritt.

Karin hingegen ist stets am Abrechnen, Gewinn und Schuld auflistend. Sie lebt in einem unendlichen Gefühl von Schuld und Reue, das sie auch zu schrecklichen Akten der Selbstbestrafung führen kann. Mit grosser Überzeugung bringt sie die radikale Gnadenlosigkeit des Lebens zum Ausdruck. Das Leben wird als eine unendliche Pflicht wahrgenommen, die nie ganz zu erfüllen ist. Damit ist sie dem Julián in Truman nicht unähnlich, nur viel verzweifelter.

Anna schließlich, die Magd, versteht ihre Aufgabe, vornehmlich ihre Pflegefunktion, als einen Liebesdienst, gegenüber den verschiedenen Familienmitgliedern, aber in erster Linie gegenüber Agnes, die sie bis am Schluss in den Tod hinein begleitet. Durch das Lesen des Tagebuches wird sie Agnes' Erbe pflegen, in einer Art gläubiger Verehrung.

\section{Zum Abschluss: eine filmische Pietà ...}

In der auf den Tod folgenden Nacht, in einer traumartigen Szene, wird die verstorbene Agnes wieder "wach« und lässt ihre zwei Schwestern zu sich rufen: Sie könne nicht sterben, es sei ihr eiskalt, sie fühle sich einsam und verlassen und sie brauche Hilfe,Wärme und Zärtlichkeit. Die zwei Schwestern schrecken davor zurück, verweigern ihr die erbetene Hilfe; die Magd hingegen bleibt bei ihr, setzt 
sich auf das Bett, nimmt sie auf ihren Schoss, legt ihr Haupt an ihre nackte Brust und schenkt ihr so die gewünschte Wärme. Das Bild der ruhig im Schoss der Magd liegenden Verstorbenen wurde zum offiziellen Filmposter. Es gleicht ganz erstaunlich einer Pietà-Szene, wie wir sie aus der Kunstgeschichte kennen: die Mutter Maria, die weinend ihren verstorbenen Sohn auf dem Schoss hält. Es könnte aber auch eine verleiblichte Darstellung dessen sein, was Spiritual Care am Lebensende bedeutet ...

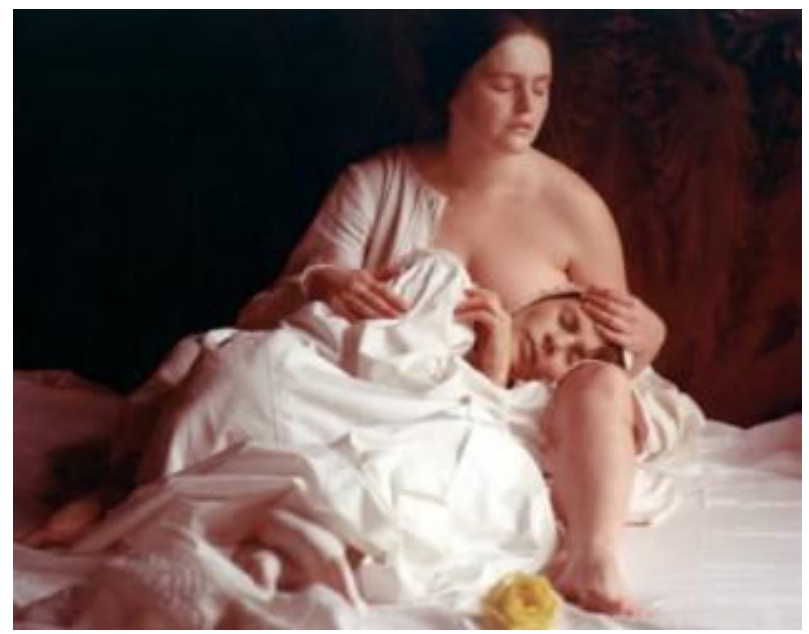

- Pierre Bühler ist emeritierter Professor für systematische Theologie, von 1982 bis 1997 an der Theologischen Fakultät der Universität Neuchâtel, von 1997 bis 2015 an der Theologischen Fakultät der Universität Zürich. 